\title{
Separation of Opiate Isomers using Electrospray Ionization and Paper Spray Coupled to High-Field Asymmetric Waveform Ion Mobility Spectrometry
}

\author{
Nicholas E. Manicke ${ }^{1}$ and Michael Belford ${ }^{2}$ \\ ${ }^{1}$ Department of Chemistry and Chemical Biology, Indiana University-Purdue University Indianapolis, Indianapolis, IN, USA \\ ${ }^{2}$ Thermo Fisher Scientific, San Jose, CA, USA \\ nmanicke@iupui.edu
}

ABSTRACT

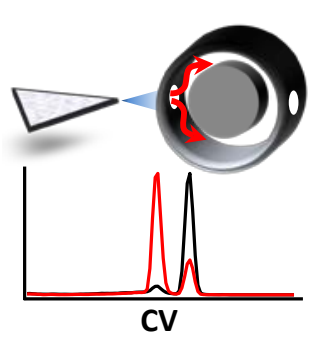

One limitation in the growing field of ambient or direct analysis methods is reduced selectivity caused by the elimination of chromatographic separations prior to mass spectrometric analysis. We explored the use of high-field asymmetric waveform ion mobility spectrometry (FAIMS), an ambient pressure ion mobility technique, to separate the closely related opiate isomers of morphine, hydromorphone, and norcodeine. These isomers cannot be distinguished by tandem mass spectrometry. Separation prior to MS analysis is therefore required to distinguish these compounds, which are important in clinical chemistry and toxicology. The FAIMS was coupled to a triple quadrupole mass spectrometer, and ionization was performed using either a pneumatically assisted heated electrospray ionization source (H-ESI) or paper spray, a direct analysis method which has been applied to the direct analysis of dried blood spots and other complex samples. We found that FAIMS was capable of separating the three opiate structural isomers using both $\mathrm{H}-\mathrm{ESI}$ and paper spray as the ionization source.

\section{Introduction}

Paper spray MS, first described in 2010, is a method for performing rapid, direct analysis of samples spotted on paper or other porous substrates $(1,2)$. A liquid sample, such as blood or urine, is spotted onto the paper and stored as a dried spot. Analysis is performed by depositing a small volume of solvent to the paper where it wicks through the porous substrate and sample by capillarity. The solvent performs the dual functions of extraction and spray ionization. An electrospray is then induced at the sharp tip of the paper following application of high voltage. Chemicals which are both soluble in the extraction/spray solvent and ionizable will be detected immediately by the mass spectrometer. The entire analysis takes about 60 seconds. Reported applications include quantitative analysis of pharmaceuticals from biofluid samples at low or sub-ng/mL levels(3-5), profiling of lipids in bacteria(6), detection of chemical contaminants in food (7), and analysis of acyl-carnitines from blood(8).

One common feature of ambient or direct MS methods, including paper spray, is the elimination of chromatography. While removing this step speeds up sample analysis and eases sample preparation constraints, it also hurts selectivity. Structurally similar constitutional isomers and diastereomers cannot be distinguished by single stage mass spectrometry and often cannot be fully distinguished even by tandem mass spectrometry.

High-field asymmetric waveform ion mobility spectrometry (FAIMS) is a gas phase separation technique that separates ions on the basis of differences in their mobilities between high and low electric fields as the ions travel through two concentric electrodes $(9,10)$. It is related to differential mobility spectrometry (DMS), which uses planar electrodes rather than cylindrical electrodes. Coupling of FAIMS with atmospheric pressure ionization (API) mass spectrometers using ESI and atmospheric pressure chemical ionization (APCI) has been widely reported. Because the time-scale of FAIMS separations is short (around 50 ms), selectivity can be improved without significantly increasing analysis time. Moreover, FAIMS interfaces with triple quadrupole and ion trap mass spectrometers better than drift tube ion mobility, although the resolution is generally poorer.

We are interested in using FAIMS to improve selectivity during paper spray MS, especially with respect to drug detection. There are a number of papers in the literature demonstrating separation of isomers by FAIMS. Most of the papers deal with peptides, including sequence isomers(11) and modification site variants. There are only a few papers describing separation of isomeric drugs or other small molecules, includingsolution of ephedra isomers(12) and leucine and isoleucine(13). There have been a few published studies reporting the combination of direct or ambient methods to differential mobility. These include the use of planar DMS to improve signal-to-noise in DESI imaging(14), 
analysis of testosterone from a solvent extract of serum using laser diode thermal desorption (LDTD) coupled to planar DMSMS/MS(15), and separation of some drugs and metabolites, including morphine glucuronide, from tissue using liquid extraction surface analysis (LESA) coupled to planar DMSMS/MS(16). A study using ESI-FAIMS-MS for measuring morphine and codeine from urine has also been reported(17).

In this study, we explored the feasibility of using electrospray and paper spray FAIMS to separate three commonly encountered and structurally similar opiate isomers: morphine, hydromorphone, and norcodeine (structures shown in supplementary figure 1). Distinguishing these isomers is important in such fields as clinical and forensic toxicology and management of chronic pain. In forensic toxicology, for example, opiate isomers must be distinguished because of the different mortality risk associated with each compound. Morphine is a strong opioid and also one of the primary metabolites of heroin. Norcodeine, on the other hand, is a metabolite of the weak opioid codeine.

\section{Experimental}

Morphine, norcodeine, and hydromorphone were purchased from Cerilliant Corporation. The spray solvents were 50:50 methanol:water with $0.1 \%$ acetic acid for heated electrospray (HESI) and 80:20 methanol:water with 0.01\% acetic acid for paper spray. A larger proportion of methanol was used for paper spray to reduce the initial droplet size and to more closely mimic normal paper spray solvents used in the analysis of dried blood, plasma, and urine spots. Thirty $\mu \mathrm{L}$ of solvent was added to the paperThe size of the paper (Whatman 31ET-Chr) was about $0.5 \mathrm{~cm}^{2}$, and 30 $\mu \mathrm{L}$ of solvent was added H-ESI conditions were as follows: spray voltage: $+3800 \mathrm{~V}$; solution flow rate: $2 \mu \mathrm{L} / \mathrm{min}$; sheath gas: 2 .

Experiments were done with a commercial FAIMS interfaced to a TSQ Vantage (Thermo Fisher, San Jose CA). The TSQ Vantage was modified for the use of either a high capacity ion transfer tube (HCTT) (18) (0.6 mm x $2.0 \mathrm{~mm}$ ellipse-like orifice) or a standard Vantage ion transfer tube (ITT) (0.6 mm diameter). Modified FAIMS electrodes designed to improve ion transfer efficiency at the atmosphere-FAIMS interface were used(18). Larger radius inner electrodes, which result in a higher electric field, were also used in the FAIMS. Additional details are provided below. The FAIMS carrier gas was nitrogen. The carrier gas flow rate was $6.0 \mathrm{~L} / \mathrm{min}$ when using the HCTT and the smaller inner FAIMS electrode. The gas flow rate was decreased to 2.0 $\mathrm{L} / \mathrm{min}$ for the standard small bore ITT and larger inner FAIMS electrode.

\section{Results and Discussion}

\section{MS/MS of morphine, norcodeine, and hydromorphone}

When analyzed by MS/MS on a triple quadrupole mass spectrometer, each of the three opiate isomers gave abundant product ions which can be used for structural identification (supplementary figure 1). There was extensive overlap between the fragment ions generated by three isomers, however, due to their highly similar chemical structures. Performing quantitative analysis or even qualitative identification from mixtures of these isomers is therefore problematic. Morphine, norcodeine, and hydromorphone can be separated by high performance liquid chromatography (HPLC)(19), but cannot be adequately distinguished when relying on MS/MS alone for selectivity.

\section{Optimization of FAIMS Separation}

In this study, we optimized the FAIMS separation conditions using direct infusion with a H-ESI source. The following FAIMS parameters were varied: FAIMS electrode temperature, internal diameter of the MS inlet capillary, and the diameter of the inner FAIMS electrode.

The temperature of the FAIMS electrodes has an impact on separation, desolvation, ion transfer efficiency, and ion mobility. The FAIMS used here can actively and independently control the temperatures of the inner and outer electrodes, which allows the user to exert some additional control over the separation. A previous study examined the impact of electrode heating in some detail(20) and found that maintaining the inner electrode at a cooler temperature than the outer electrode results in narrower peaks at lower compensation voltages (CV), but with lower signal intensities, due to changes in the focusing fields within the device.

Figure 1 shows the CV scans for a $500 \mathrm{ng} / \mathrm{mL}$ mixture of norcodeine and hydromorphone analyzed via direct infusion with H-ESI in SRM mode at two different electrode temperature settings: the inner and outer electrode both at $100^{\circ} \mathrm{C}$ (Figure 1A) and with the inner electrode at $70^{\circ} \mathrm{C}$ while holding the outer electrode at $100^{\circ} \mathrm{C}$ (Figure 1B). The larger bore HCTT was used

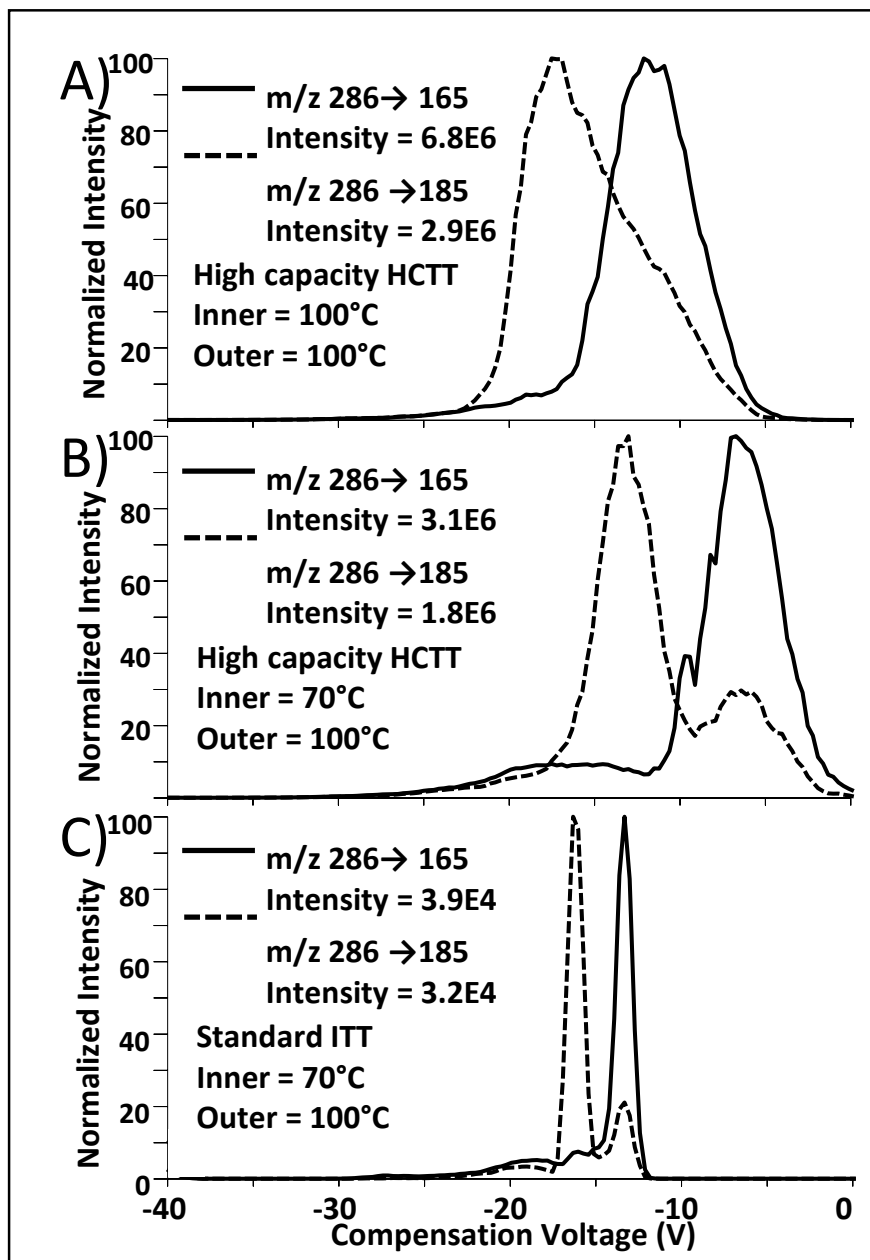

Figure 1. FAIMS CV scans for a mixture of hydromorphone and norcodeine. CV scans $A$ and $B$ were collected using the larger bore HCTT with either (A) both electrodes at $100^{\circ} \mathrm{C}$ or (B) the inner electrode at $70^{\circ} \mathrm{C}$ and the outer electrode at $100^{\circ} \mathrm{C}$. In (C), the standard smaller bore ITT was used. The electrode temperatures were the same as in (B). 
and the gap between the inner and outer FAIMS electrode was $1.5 \mathrm{~mm}$. Due to the overlap in the fragment ions generated by hydromorphone and norcodeine, both SRM channels shown here should have two peaks when the two components in the mixture are resolved. With both electrodes at $100^{\circ} \mathrm{C}$, separate peaks for the two components were not observed. The peaks from the two SRM channels are noticeably shifted due to the differences in relative intensity of the fragment ions generated by the two compounds. By decreasing the temperature of the inner electrode, the peaks in the CV scan became narrower and shifted to lower $\mathrm{CV}$ values. In addition, separate peaks for hydromorphone and norcodeine became observable. Separate infusion of the pure compounds showed that hydromorphone was responsible for the peak at higher (more negative) $\mathrm{CV}$ values while norcodeine gave the peak at lower CV values. Cooling the inner electrode did however decrease ion intensity, consistent with previous work(20).

The size of the MS inlet opening has a significant effect on both the FAIMS separation and the signal intensity. A higher throughput inlet capillary (accompanied by a high capacity pumping system) tends to improve ion transfer efficiency and increase MS signal intensity. A larger bore MS inlet negatively impacts separation in the FAIMS, however, because the ion residence time in the FAIMS is decreased due to the higher carrier gas velocity(20). The CV scans in Figure $1 \mathrm{~A}$ and $\mathrm{B}$ were collected using the larger bore HCTT, while Figure $1 \mathrm{C}$ was collected using the smaller bore ITT. Going to the smaller diameter ITT significantly increased the separation efficiency of the FAIMS as shown by the narrower peaks and better resolution between hydromorphone and norcodeine. Increasing the size of the

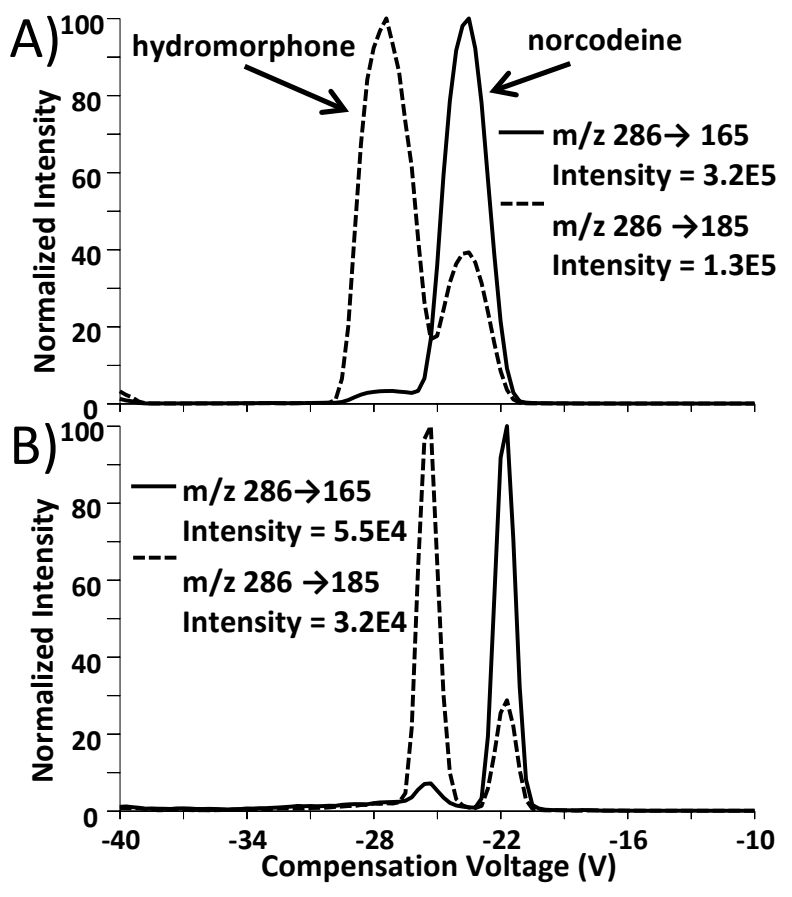

Figure 2. FAIMS CV scans for a mixture of hydromorphone and norcodeine collected using the standard small bore ITT and a smaller FAIMS electrode gap ( $1.25 \mathrm{~mm}$ compared to $1.5 \mathrm{~mm}$ gap used in Figure 1). A) both inner electrode and outer electrode set a $100^{\circ} \mathrm{C}$. B) inner electrode $=70^{\circ} \mathrm{C}$, outer electrode $=100^{\circ} \mathrm{C}$. Note the smaller range of the $\mathrm{CV}$ scan compared to Figure 1. inner FAIMS electrode while holding the size of the outer electrode constant decreases the annular gap through which the ions travel and increases the magnitude of the electric field at a given dispersion voltage(21). This is known to give narrower peaks in the FAIMS CV scan and shift peaks to higher CV values. FAIMS CV scans using a smaller gap of $1.25 \mathrm{~mm}$ (compared to $1.5 \mathrm{~mm}$ in the previous experiments) between the inner and outer electrode at different temperature settings for a mixture of hydrocodone and norcodeine are shown in Figure 2. By decreasing the gap between the FAIMS electrodes, using the smaller bore ITT, and holding the inner electrode at a lower temperature than the outer electrode, baseline resolution between hydromorphone and norcodeine was achieved without any decrease in MS signal intensity (Figure 2B).

A mixture containing all three opiate isomers (morphine, hydromorphone, and norcodeine) was then analyzed by direct infusion with H-ESI using the same FAIMS conditions as in Figure 2B. There is extensive overlap in the fragment ions generated by these three compounds. As a result, all SRM channels contain contributions from multiple compounds. By setting FAIMS parameters to maximize resolution, all three structural isomers could be resolved as seen in Figure 4A. By setting the $\mathrm{CV}$ to -25.6 , -23.7 or, $-22.1 \mathrm{~V}$, interference free MS/MS spectra were obtained for hydromorphone, morphine, and norcodeine, respectively. Interference was evaluated by comparing relative fragment intensities obtained from infusion of the pure compounds to the relative fragment intensities obtained from the opiate mixture separated by FAIMS prior to MS/MS.

\section{Paper spray}

Paper spray was coupled to the FAIMS-MS/MS by removing the commercial H-ESI probe and positioning a prototype paper spray cartridge in front of the FAIMS inlet (supplementary figure 2). The cartridge consisted of a small block of acetal with a slot machined into one end to hold the paper substrate and a hole bored through the top surface for solvent application. A wire contacting the paper substrate was connected to the spray voltage power supply on the mass spectrometer.

Figure 4B shows the separation of the three opiate isomers using paper spray as the ion source rather than H-ESI. The opiate isomers were dissolved in the spray solvent at a concentration of 5 $\mu \mathrm{g} / \mathrm{mL}$. The quality of the separation obtained via paper spray was similar to that obtained from H-ESI in that the peak width and $\mathrm{CV}$ for each component were the same. We observed, however, that the signal from paper spray was generally lower than obtained with $\mathrm{H}-\mathrm{ESI}$, and the quality of the CV scan was less reproducible. We attributed this to poorer desolvation in paper spray compared to H-ESI. Poorer desolvation is expected to decrease ion transmission and produce extraneous or, at times, broader peaks, which is consistent with what we observed.

In H-ESI, desolvation can be improved by using sheath gas, a heated auxiliary gas, and by the counter-current gas emerging from the FAIMS entrance. We could not make use of these gas flows during paper spray because they tended to quickly dry out the paper. There was no sheath gas or heated auxillary gas during paper spray analysis. The FAIMS carrier gas flow rate was also decreased to minimize the amount of gas flowing out of the FAIMS entrance (decreased to $1.5 \mathrm{~L} / \mathrm{min}$ from the $2.0 \mathrm{~L} / \mathrm{min}$ used for H-ESI). This was required to prevent the paper substrate from quickly drying out. In paper spray MS without FAIMS, 


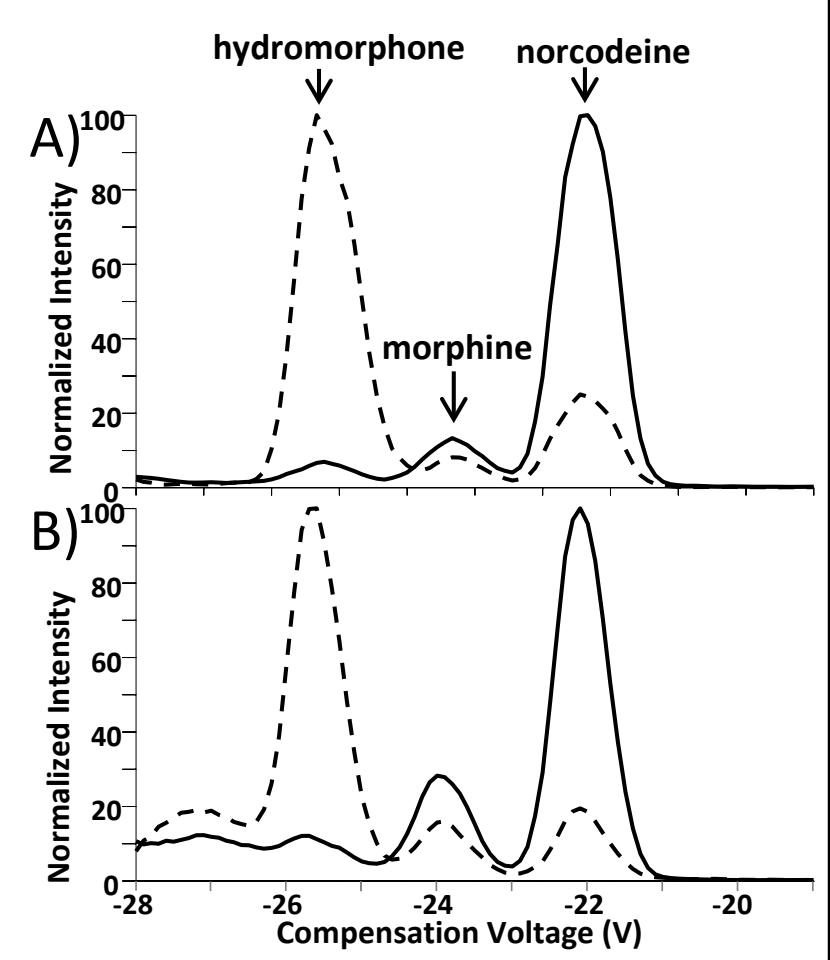

Figure 3. FAIMS CV scans for a mixture of hydromorphone, morphine, and norcodeine collected using (A) H-ESI and (B) paper spray. Dashed line: $\mathrm{m} / \mathrm{z} 286$ $\rightarrow 185$; solid line: $\mathrm{m} / \mathrm{z} 286 \rightarrow 165$. Note the smaller CV scan range compared to Figure 1 and Figure 2.

desolvation occurs at the heated atmospheric pressure inlet of the mass spectrometer. Because the heated inlet is located after the FAIMS apparatus, it could not be relied on for desolvation in this experiment. The situation with paper spray is further complicated in that the droplet size and solvent flow rate varies as a function of time as the spray solvent is depleted during the analysis(22). To improve desolvation in these experiments, the paper spray tip was moved about $1.5 \mathrm{~cm}$ farther back than is typical and positioned off-axis.

\section{Conclusion}

FAIMS shows good potential for improving selectivity in mass spectrometric analyses. This is likely to be particularly valuable for direct or ambient ionization methods such as paper spray that omit chromatographic separations in order to increase throughput, decrease sample preparation, and decrease the complexity of MS analysis.

Separation of the structurally similar opiate isomers morphine, norcodeine, and hydromorphone was achieved, but required configuring the FAIMS for maximum resolution (increasing ion residence time, decreasing inner electrode temperature, and decreasing the gap between the FAIMS electrodes). While these steps improved resolution, they also decreased ion intensity. Use of gas phase modifiers in the carrier gas will be explored in the future. Gas-phase modifiers in FAIMS were recently shown to improve ion signal by one to two orders of magnitude(23) and can also improve selectivity compared to unmodified carrier gases.

Coupling of paper spray to FAIMS-MS was demonstrated, and good separation of the three opiate isomers was obtained. The signal intensity and stability of paper spray was worse than H-ESI, however, which we attributed to poorer desolvation. Future research efforts therefore will focus on improving analyte desolvation in paper spray-FAIMS.

\section{Acknowledgments}

Thanks to Mari Prieto Conaway for helpful discussions and review of the manuscript

\section{References}

1. Liu J, Wang H, Manicke NE, Lin J-M, Cooks RG, Ouyang Z. Development, characterization, and application of paper spray ionization. Analytical Chemistry 2010;82:2463-71.

2. Wang H, Liu J, Cooks RG, Ouyang Z. Paper spray for direct analysis of complex mixtures using mass spectrometry. Angewandte Chemie International Edition 2010;49:877-80.

3. Manicke NE, Yang Q, Wang H, Oradu S, Ouyang Z, Cooks RG. Assessment of paper spray ionization for quantitation of pharmaceuticals in blood spots. International Journal of Mass Spectrometry 2011;300:123-9.

4. Manicke NE, Abu-Rabie P, Spooner N, Ouyang Z, Cooks RG. Quantitative analysis of therapeutic drugs in dried blood spot samples by paper spray mass spectrometry: An avenue to therapeutic drug monitoring. Journal of the American Society for Mass Spectrometry 2011;22:1501-7.

5. Shi R-Z, El Gierari ETM, Manicke NE, Faix JD. Rapid measurement of tacrolimus in whole blood by paper spray-tandem mass spectrometry (ps-ms $/ \mathrm{ms}$ ). Clinica Chimica Acta 2015;441:99-104.

6. Hamid AM, Jarmusch AK, Pirro V, Pincus DH, Clay BG, Gervasi G, Cooks RG. Rapid discrimination of bacteria by paper spray mass spectrometry. Analytical Chemistry 2014;86:7500-7.

7. Zhang ZP, Cooks RG, Ouyang Z. Paper spray: A simple and efficient means of analysis of different contaminants in foodstuffs. Analyst 2012;137:2556-8.

8. Yang Q, Manicke NE, Wang H, Petucci C, Cooks RG, Ouyang Z. Direct and quantitative analysis of underivatized acylcarnitines in serum and whole blood using paper spray mass spectrometry. Anal Bioanal Chem 2012;404:1389-97. 9. Buryakov IA, Krylov EV, Nazarov EG, Rasulev UK. A new method of separation of multi-atomic ions by mobility at atmospheric-pressure using a highfrequency amplitude-asymmetric strong electric-field. International Journal of Mass Spectrometry and Ion Processes 1993;128:143-8.

10. Purves RW, Guevremont R, Day S, Pipich CW, Matyjaszczyk MS. Mass spectrometric characterization of a high-field asymmetric waveform ion mobility spectrometer. Review of Scientific Instruments 1998;69:4094-105.

11. Shvartsburg AA, Creese AJ, Smith RD, Cooper HJ. Separation of a set of peptide sequence isomers using differential ion mobility spectrometry. Analytical Chemistry 2011;83:6918-23.

12. McCooeye M, Ding L, Gardner GJ, Fraser CA, Lam J, Sturgeon RE, Mester Z. Separation and quantitation of the stereoisomers of ephedra alkaloids in natural health products using flow injection-electrospray ionization-high field asymmetric waveform ion mobility spectrometry-mass spectrometry. Analytical Chemistry 2003;75:2538-42.

13. Barnett DA, Ells B, Guevremont R, Purves RW. Separation of leucine and isoleucine by electrospray ionization-high field asymmetric waveform ion mobility spectrometry-mass spectrometry. Journal of the American Society for Mass Spectrometry 1999;10:1279-84.

14. Bennett RV, Gamage CM, Galhena AS, Fernandez FM. Contrast-enhanced differential mobility-desorption electrospray ionization-mass spectrometry imaging of biological tissues. Analytical Chemistry 2014;86:3756-63.

15. Picard P, Lacoursiere J, Tremblay P, Jarvis M, Ghobarah H, Taylor A. Selectivity enhancement in high throughput analysis of testosterone using differential ion mobility coupled to ldtd-ms/ms. Mass Spectrometry: Applications to the Clinical Laboratory, Vol. San Diego CA, 2012.

16. Porta T, Varesio E, Hopfgartner G. Gas-phase separation of drugs and metabolites using modifier-assisted differential ion mobility spectrometry hyphenated to liquid extraction surface analysis and mass spectrometry. Analytical Chemistry 2013;85:11771-9.

17. McCooeye MA, Ells B, Barnett DA, Purves RW, Guevremont R. Quantitation of morphine and codeine in human urine using high-field asymmetric waveform ion mobility spectrometry (faims) with mass spectrometric detection. Journal of Analytical Toxicology 2001;25:81-7.

18. Prasad S, Belford MW, Dunyach J-J, Purves RW. On an aerodynamic mechanism to enhance ion transmission and sensitivity of faims for nanoelectrospray ionization-mass spectrometry. Journal of the American Society for Mass Spectrometry 2014;25:2143-53.

19. Edinboro LE, Backer RC, Poklis A. Direct analysis of opiates in urine by liquid chromatography-tandem mass spectrometry. Journal of Analytical Toxicology 2005;29:704-10

20. Barnett DA, Belford M, Dunyach J-J, Purves RW. Characterization of a 
temperature-controlled faims system. Journal of the American Society for Mass Spectrometry 2007;18:1653-63.

21. Barnett DA, Ouellette RJ. Elimination of the helium requirement in high-field asymmetric waveform ion mobility spectrometry (faims): Beneficial effects of decreasing the analyzer gap width on peptide analysis. Rapid Communications in Mass Spectrometry 2011;25:1959-71.

22. Espy RD, Muliadi AR, Ouyang Z, Cooks RG. Spray mechanism in paper spray ionization. International Journal of Mass Spectrometry 2012;325:167-71.

23. Purves RW, Ozog AR, Ambrose SJ, Prasad S, Belford M, Dunyach JJ. Using

gas modifiers to significantly improve sensitivity and selectivity in a cylindrical

faims device. Journal of the American Society for Mass Spectrometry

2014;25:1274-84 